01

\title{
О выборе дистрибутивного базиса в расчетах дипольных моментов перехода в однодетерминантном приближении
}

\author{
(C) В.Н. Глушков, С.И. Фесенко
}

Днепропетровский национальный университет им. О. Гончара, 49010 Днепропетровск, Украина

e-mail: v_n_glushkov@yahoo.com

Поступила в редакцию 27.12.2017 г.

В окончательной редакции 12.02.2018 г.

\begin{abstract}
Представлена попытка применения так называемой методологии асимптотического проектирования, развитой нами ранее, к вычислению электронных дипольных моментов перехода (ДМП) в однодетерминантном приближении между состояниями с одинаковой спиновой и пространственной симметрией. Кратко обсуждены основные уравнения метода и особенности их применения к расчету ДМП. При этом внимание акцентировано на выборе конечного одночастичного базиса. Для этого предложены два возможных алгоритма построения дистрибутивных базисов. Первый из них для построения использует минимизацию энергии Хартри-Фока $\left(E_{X \Phi}\right)$ по нелинейным параметрам базиса. Во втором случае параметры определяются минимизацией функционала $E=E_{\mathrm{X} \Phi}+E_{\mathrm{MP} 2}$, который включает электронную корреляцию через второй порядок теории возмущений Меллера-Плесета $\left(E_{\mathrm{MP2}}\right)$. Результаты вычислений в обоих базисах в целом демонстрируют согласие с высокоточными расчетами, выполненными методами конфигурационного взаимодействия. При этом базис, адаптированный для $E=E_{\mathrm{X \Phi}}+E_{\mathrm{MP} 2}$, существенно улучшает точность расчета по сравнению с базисом первого типа.
\end{abstract}

DOI: $10.21883 /$ OS.2018.06.46073.307-17

\section{Введение}

Современные многочастичные методы расчета электронной структуры молекул являются настолько изощренными, что основным источником ошибок в неэмпирических вычислениях становится неизбежная погрешность, связанная с конечностью используемого одночастичного базиса. Конечность базисов приводит к тому, что базисы, оптимальные для определения, например энергии, не являются хорошим приближением для расчета других свойств, например поляризуемости молекул, силовых постоянных и т.п. В частности, даже в случае расчета энергии необходимо различать критерии в построении базиса для энергии в приближении Хартри-Фока (ХФ) и для корреляционной энергии. Хорошо, если для выбора базисов можно использовать вариационные принципы, приспособленные для расчета заданной величины. Тогда можно ожидать, что последовательность увеличивающихся по размеру базисов обеспечивает сходимость значений искомой величины к пределу полного базиса. Например, для энергии ХФ такая последовательность оптимальных базисов строится минимизацией частного Релея по параметрам базиса [1-4]. Базис, оптимальный для энергии корреляции во втором порядке многочастичной теории возмущений, может быть выбран путем минимизации функционала Хиллераса [5]. Существуют и другие полезные вариационные оценки [6,7].

Однако для расчета большинства физических характеристик отсутствует их непосредственное сопоставление некоему вариационному принципу. В частности, это относится к дипольным моментам перехода (ДМП), знание которых составляет основу определения важных спектроскопических характеристик, таких как силы осцилляторов, вероятности перехода, время жизни состояний и др. По своему определению ДМП зависят от волновых функций $\Psi_{a}$ и $\Psi_{b}$, отвечающих различным состояниям:

$$
\mu_{a b}=\left\langle\Psi_{a}|\boldsymbol{\mu}| \Psi_{b}\right\rangle
$$

где $\boldsymbol{\mu}$ - оператор дипольного момента. Поэтому они очень чувствительны к точности определения $\Psi_{a}$ и $\Psi_{b}$ и, следовательно, к выбору базиса, который, вообще говоря, должен быть различным для основного и возбужденного состояний (ВС). На привлекательность такого подхода указывалось еще в работе [8].

Конечно, для современных практических приложений предпочтительней оказываются расчеты, основанные на различных вариантах методов конфигурационного взаимодействия, которые используют несколько опорных конфигураций (так называемые multireference configuration interaction (MR-CI) methods) [9-15]. Так, например, в работе [15] вычисления проводились в пространстве, состоящем из 11.8 миллионов конфигураций. Значения теоретических времен жизни возбужденных состояний молекулы CN отличались от экспериментальных в рамках 5\%, что сравнимо с погрешностью самого эксперимента. К сожалению, подобные вычисления не стали пока рутинными из-за трудностей их практической реализации.

Методы, использующие приближение ХФ в расчетах ДМП, представлены в литературе в меньшей степени. Они, как правило, рассматривают переходы между 
состояниями с различной пространственной симметрией [16]. Насколько нам известно, практически отсутствуют работы, где исследуются возможности однодетерминантного приближения для расчета молекулярных ДМП между состояниями одинаковой пространственной и спиновой симметрии. Причиной тому, в частности, являются проблемы, связанные с эффективным обеспечением ортогональности состояний в приближении ХФ и выбором одночастичного базиса, адаптированного для ДМП [17-22].

В настоящей работе на примере молекулы НеН представлена попытка восполнить данный пробел, акцентируя внимание на выборе конечного базиса для расчета ДМП и применяя ранее предложенную нами методологию асимптотического проектирования (АП) для обеспечения ортогональности состояний [22]. Для этого мы исследуем различные возможности оптимизации одночастичного базиса. В первом варианте нелинейные параметры гауссова базиса определяются минимизацией энергии ХФ $\left(E_{Х \Phi}\right)$ для каждого индивидуального состояния. Второй вариант рассматривает возможность выбора базиса путем минимизации энергии, определенной как сумма $E=E_{\mathrm{X} \Phi}+E_{\mathrm{MP} 2}$ (где $E_{\mathrm{MP} 2}-$ корреляционная энергия, вычисленная во втором порядке теории возмущений (ТВ) Меллера-Плессета (МП)). При этом ТВМП, предложенная ранее для основного состояния [23], была нами модифицирована для ВС одинаковой симметрии (см.также [22]). Построенные таким образом базисы используются для расчета ДМП между состояниями $\mathrm{A}^{2} \Sigma^{+} \rightarrow \mathrm{X}^{2} \Sigma^{+}, \mathrm{C}^{2} \Sigma^{+} \rightarrow \mathrm{X}^{2} \Sigma^{+}$и $\mathrm{D}^{2} \Sigma^{+} \rightarrow \mathrm{X}^{2} \Sigma^{+}$в однодетерминантном приближении.

\section{Метод}

В этом разделе рассматриваются уравнения ХФ, определяющие орбитали ВС, и соответствующие выражения для энергии корреляции во втором порядке многочастичной теории возмущений. Детали вывода уравнений можно найти в наших предыдущих работах [22,24-26]. Поэтому ограничиваемся формулами, на основе которых были проведены расчеты.

Так, например, модифицированные уравнения ХФ для орбиталей $\varphi_{i}^{\sigma}$ первого ВС в конечном базисе $\left\{\chi_{p}\right\}_{1}^{M}$

$$
\varphi_{i}^{\sigma}=\sum_{p=1}^{M} c_{p i}^{\sigma} \chi_{p}, \quad \sigma=\alpha, \beta
$$

имеют вид

$$
F_{\bmod }^{\sigma} \mathbf{c}_{i}^{\sigma}=\varepsilon_{i}^{\sigma} S \mathbf{c}_{i}^{\sigma},
$$

где $\mathbf{c}_{i}^{\sigma}$ - векторы-столбцы из коэффициентов $c_{p i}^{\sigma}, S-$ матрица перекрывания с элементами $S_{p q}=\left\langle x_{p} \mid \chi_{q}\right\rangle$ и $F_{\text {mod }}^{\sigma}$ - модифицированные матрицы Фока:

$$
\begin{gathered}
F_{\text {mod }}^{\alpha}=F^{\alpha}-\lambda_{s} S P^{\beta} S+\lambda_{0} S P_{0}^{\alpha} S, \\
F_{\text {mod }}^{\beta}=F^{\beta}+\lambda_{s} S Q^{\alpha} S .
\end{gathered}
$$

Здесь $F^{\alpha}$ и $F^{\beta}$ - стандартные матрицы неограниченного ХФ (НХФ) метода с элементами $F_{p q}^{\alpha}=\left\langle\chi_{p}\left|F^{\alpha}\right| \chi_{q}\right\rangle$ и $F_{p q}^{\beta}=\left\langle\chi_{p}\left|F^{\beta}\right| \chi_{q}\right\rangle, P^{\beta}=\sum_{i=1}^{n^{\beta}} \mathbf{c}_{i}^{\beta}\left(\mathbf{c}_{i}^{\beta}\right)^{T}$ - матрица плотности из занятых орбиталей $\beta$-роя, $Q^{\alpha}=\sum_{i=n^{\alpha}+1}^{M} \mathbf{c}_{i}^{\alpha}\left(\mathbf{c}_{i}^{\alpha}\right)^{T}-$ матрица плотности из виртуальных орбиталей $\alpha$ роя $\quad$ и $\quad P_{0}^{\alpha}=\mathbf{c}_{0 n}^{\alpha}\left(\mathbf{c}_{0 n}^{\alpha}\right)^{T} \quad$ - матрица ортопроектора $P_{0}^{\alpha}=\left|\varphi_{0 n}^{\alpha}\right\rangle\left\langle\varphi_{0 n}^{\alpha}\right|$ на подпространство высшей по энергии орбитали $\varphi_{0 n}^{\alpha}$ в детерминанте основного состояния

$$
\Phi_{0}=\frac{1}{\sqrt{N !}} \operatorname{det}\left|\varphi_{01^{\alpha}} \alpha, \ldots, \varphi_{0 n^{\alpha}} \alpha ; \varphi_{01^{\beta}} \beta, \ldots, \varphi_{0 n^{\beta}} \beta\right| .
$$

Для описания первого ВС той же симметрии используется детерминант $\Phi_{1}$

$$
\Phi_{1}=\frac{1}{\sqrt{N !}} \operatorname{det}\left|\varphi_{1^{\alpha}} \alpha, \ldots, \varphi_{n^{\alpha}} \alpha ; \varphi_{1^{\beta}} \beta, \ldots, \varphi_{n^{\beta}} \beta\right|,
$$

где $n^{\alpha}+n^{\beta}=N$ - число электронов, $n^{\alpha}>n^{\beta}, n^{\alpha}-n^{\beta}=$ $=2 S=2 S_{z}, S-$ спин системы.

Как видно, уравнения для ВС (3)-(5) отличаются от традиционных уравнений НХФ для основного состояния легко вычисляемыми добавками (типа элементов перекрывания). В соответствии с методологией асимптотического проектирования [22,24-27] слагаемые при множителе $\lambda_{s}$ обеспечивают спиновую чистоту, тогда как слагаемые при $\lambda_{0}$ ведут к ортогональности функций (6) и (7), т.е. $\left\langle\Phi_{0} \mid \varphi_{1}\right\rangle=0$. Указанные требования выполняются в пределе $\lambda_{0}, \lambda_{s} \Rightarrow \infty$ (на практике значения $\lambda_{s}=100$ a.u. и $\lambda_{0}=1000$ a.u. обеспечивают заданную точность). Найденные таким образом орбитали ВС позволяют вычислить энергию в рамках стандартного формализма НХФ. Полезно отметить, что вышеуказанные уравнения предусматривают возможность использования различных базисов для разных состояний. В этом случае матрица перекрывания строится на функциях, принадлежащих разным базисным наборам.

Аналогия уравнений (3) с уравнениями метода НХФ непосредственно ведет к построению теории возмущений Меллера-Плесета для учета корреляционных поправок ВС. Так, например, поправка второго порядка к энергии первого ВС имеет вид $[24,26]$

$$
\begin{aligned}
E_{\mathrm{MP} 2}= & \sum_{i>j}^{\text {occ }} \sum_{a>b}^{\text {virt }} \frac{\left|\left(\varphi_{a} \varphi_{i} \mid \varphi_{b} \varphi_{j}\right)-\left(\varphi_{a} \varphi_{j} \mid \varphi_{b} \varphi_{i}\right)\right|^{2}}{\varepsilon_{i}+\varepsilon_{j}-\varepsilon_{a}-\varepsilon_{b}} \\
& -\left\langle\Phi_{1}|H| \Phi_{0}\right\rangle\left\langle\Phi_{0}^{(1)} \mid \Phi_{1}\right\rangle .
\end{aligned}
$$

Здесь нижние индексы $i$ и $j$ используются для занятых орбиталей в детерминанте $\Phi_{1}$, а индексы $a, b$ - для виртуальных обриталей. Суммирование в (8) проводится по спин-орбиталям. Через $\Phi_{0}^{(1)}$ обозначена поправка первого порядка к волновой функции основного состояния.

Как видно, первое слагаемое в (8) соответствует выражению энергии второго порядка ТВМП для основного состояния. Появление второго слагаемого обусловлено учетом ортогональности и тем фактом, что функции 
ХФ не являются собственными для гамильтониана $H$. Можно ожидать, что если волновые функции $\Phi_{0}$ и $\Phi_{1}$ определены достаточно точно [26], то матричный элемент $\left\langle\Phi_{1}|H| \Phi_{0}\right\rangle$ будет мал. Более того, элемент перекрывания $\left\langle\Phi_{0}^{(1)} \mid \Phi_{1}\right\rangle<1$. Поэтому на данной стадии вычислений мы пренебрегали вторым слагаемым в (8).

Таким образом, применение техники АП для учета ортогональности ведет к тому, что компьютерные затраты для ВС на уровне вычисления энергий $E_{\mathrm{X} \Phi}$ и $E_{\mathrm{X} \Phi}+E_{\mathrm{MP} 2}$ практически сравнимы с соответствующими затратами для основного состояния.

\section{Алгоритм и обсуждение результатов расчета}

Молекула НеН является одной из простых молекул, для расчета которых возможно использовать достаточно точные волновые функции, построенные из огромного числа конфигураций. Для нее, в частности, было проведено теоретическое изучение ДМП, используя для этого различные методы [9-12]. Результаты таких работ можно рассматривать как эталонные и использовать их в качестве тестирования работоспособности новых методов.

Ниже в однодетерминантном приближении будут представлены результаты расчетов ДМП между $\Sigma^{+}$-состояниями молекулы НеH, полученные в базисах, которые адаптированы для энергии в приближении ХФ $\left(E_{\mathrm{X} \Phi}\right)$, и в базисах, которые минимизируют энергию во втором порядке ТВ $\left(E_{\mathrm{X} \Phi}+E_{\mathrm{MP} 2}\right)$. Наши расчеты были выполнены в базисах из гауссовых сферических функций:

$$
\begin{gathered}
\chi_{p}(x, y, z)=\exp \left\{-\varsigma_{p}\left[x^{2}+y^{2}+\left(z-Z_{p}\right)^{2}\right]\right\}, \\
p=1,2, \ldots, M
\end{gathered}
$$

которые распределены вдоль молекулярной оси $O Z$ (дистрибутивные базисы), $M-$ размерность базиса. Вариационные параметры (параметры экспонент $\varsigma_{p}$ и параметры центровки $Z_{p}$ ) определялись минимизацией соответствующего функционала. Отметим, что, несмотря на простоту таких функций, они оказались полезными при расчетах состояний $\Sigma$-симметрии ряда малых молекул: $\mathrm{H}_{2}, \mathrm{HeH}, \mathrm{BeH}$ и ВН. Значения энергии ХФ в таких оптимизированных базисах отличаются от ХФ-предела лишь на десятые доли микрохартри ( $\mu$ hartree) $[3,4]$.

Как отмечалось, нами рассматривались два типа базисов. В первом случае вариационные параметры находились из условия

$$
\frac{\partial E_{X \Phi}}{\partial \zeta_{p}}=0, \quad \frac{\partial E_{X \Phi}}{\partial Z_{p}}=0, \quad p=1,2, \ldots, M .
$$

Таблица 1. Полные энергии (в hartree) основного $\mathrm{X}^{2} \Sigma^{+}$ и $\mathrm{BC}^{2} \Sigma^{+}$молекулы НеН при межъядерном расстоянии $R=1.5 \mathrm{bohr}$

\begin{tabular}{l|c|c|c|c}
\hline Метод & $\mathrm{X}^{2} \Sigma^{+}$ & $\mathrm{A}^{2} \Sigma^{+}$ & $\mathrm{C}^{2} \Sigma^{+}$ & $\mathrm{D}^{2} \Sigma^{+}$ \\
\hline $\mathrm{A}^{*}$ & -3.219276 & -3.066567 & -3.014084 & -2.988346 \\
$\mathrm{~B}^{*}$ & - & -3.065836 & -3.013555 & -2.987931 \\
$\mathrm{~TB}^{* *}$ & -3.240484 & -3.090700 & -3.037288 & -3.011597 \\
$\mathrm{~KB}^{* * *}$ & -3.263173 & -3.112706 & -3.055797 & -3.030025
\end{tabular}

Примечание. *Энергии, вычисленные в однодетерминантном приближении: А - в базисе, подстроенном под $E_{\mathrm{X \Phi}}, \mathrm{B}-$ в базисе, подстроенном под $E_{\mathrm{X} \Phi}+E_{\mathrm{MP} 2}$.

** С учетом энергии корреляции, рассчитанной во втором порядке ТВ. *** Метод конфигурационного взаимодействия [11].

Для построения второго типа базисов использовались уравнения

$$
\begin{aligned}
\frac{\partial\left(E_{\mathrm{X} \Phi}+E_{\mathrm{MP} 2}\right)}{\partial \zeta_{p}} & =0, \quad \text { и } \quad \frac{\partial\left(E_{\mathrm{X} \Phi}+E_{\mathrm{MP} 2}\right)}{\partial Z_{p}}=0, \\
p & =1,2, \ldots, M .
\end{aligned}
$$

Выражения для матричных элементов одночастичного оператора дипольного момента между различными детерминантными функциями были взяты из монографии [28]. При этом стоит отметить, что соответствующие выражения являются общими и учитывают вклад неортогональных орбиталей основного и возбужденных состояний. Расчеты ДМП для НеН были проведены в базисах различной размерности $(12 s, 18 s$ и $24 s)$. Результаты показали, что базис из $18 s$-функций (9) представляет компромисс между точностью и затраченным компьютерным временем. Дальнейшие результаты будут приведены для базиса $18 s$-функций. Алгоритм вычислений заключался в следующем.

1. Для фиксированного межъядерного расстояния строим начальное приближение для параметров базиса, $\varsigma_{p}$ и $Z_{p}$. Для этого используется так называемая ангармоническая модель для построения дистрибутивных базисов [4].

2. Дальнейшая минимизация энергии $E_{X \Phi}$ методом прямого поиска (Хука-Дживса) приводит к определению нелинейных параметров, которые определяют базис. Линейные параметры $c_{p i}^{\sigma}$ определяются решением секулярного уравнения (3). Соответствующая процедура выполняется для каждого состояния.

3. Используя построенные базисы, проводится расчет ДМП.

4. Второй тип базиса определяется минимизацией энергии $E_{\mathrm{X} \Phi}+E_{\mathrm{MP2}}$. При этом в качестве нулевого приближения используется базис, адаптированный для $E_{\mathrm{X \Phi}}$. Остальные шаги выполняются в соответствии с п. 2, 3 .

В первой колонке табл. 1 указан метод, который был использован для вычисления полной энергии. Методы А и В соответствуют однодетерминантному приближению. „А“ означает, что использовался базис, который определялся минимизацией $E_{X \Phi}$. Тогда как „В“ означает, 


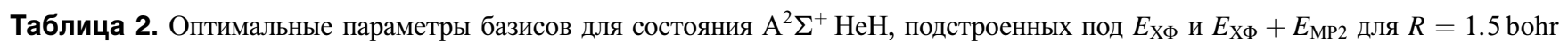

\begin{tabular}{r|c|c|c|c}
\hline \multirow{2}{*}{$p$} & \multicolumn{2}{|c|}{$\mathrm{A}^{*}$} & & $\mathrm{~S}_{p}$ \\
\cline { 2 - 5 } & $S_{p}$ & $Z_{p}$ & $Z_{p}$ \\
\hline 1 & $1.315726 \cdot 10^{3}$ & $-2.534534 \cdot 10^{-11}$ & $5.566733 \cdot 10^{2}$ & $-9.715469 \cdot 10^{-12}$ \\
2 & $1.990499 \cdot 10^{2}$ & $-1.376379 \cdot 10^{-7}$ & $7.715222 \cdot 10^{1}$ & $-5.289999 \cdot 10^{-9}$ \\
3 & $4.564899 \cdot 10^{1}$ & $4.415726 \cdot 10^{-5}$ & $1.681126 \cdot 10^{1}$ & $2.716211 \cdot 10^{-5}$ \\
4 & $1.296205 \cdot 10^{1}$ & $3.259182 \cdot 10^{-3}$ & $4.584933 \cdot 10^{0}$ & $1.138282 \cdot 10^{-3}$ \\
5 & $4.187099 \cdot 10^{0}$ & $6.547944 \cdot 10^{-3}$ & $1.426065 \cdot 10^{0}$ & $2.295554 \cdot 10^{-4}$ \\
6 & $1.644418 \cdot 10^{0}$ & $2.373382 \cdot 10^{-1}$ & $4.634538 \cdot 10^{0}$ & $1.021454 \cdot 10^{-1}$ \\
7 & $1.441474 \cdot 10^{0}$ & $-2.663461 \cdot 10^{-1}$ & $4.754496 \cdot 10^{-1}$ & $-2.214475 \cdot 10^{-2}$ \\
8 & $1.504212 \cdot 10^{0}$ & $9.650848 \cdot 10^{-1}$ & $1.398138 \cdot 10^{0}$ & $7.341389 \cdot 10^{-2}$ \\
9 & $5.441910 \cdot 10^{-1}$ & $1.299691 \cdot 10^{-1}$ & $1.026126 \cdot 10^{-1}$ & $6.746428 \cdot 10^{-1}$ \\
10 & $2.155257 \cdot 10^{-1}$ & $4.346987 \cdot 10^{-2}$ & $2.731307 \cdot 10^{-2}$ & $1.528780 \cdot 10^{-3}$ \\
11 & $5.001864 \cdot 10^{-2}$ & $-2.882917 \cdot 10^{-3}$ & $1.183878 \cdot 10^{-1}$ & $-1.016999 \cdot 10^{-4}$ \\
12 & $7.059057 \cdot 10^{1}$ & $1.496599 \cdot 10^{0}$ & $1.259149 \cdot 10^{1}$ & $1.478694 \cdot 10^{0}$ \\
13 & $1.084395 \cdot 10^{1}$ & $1.479309 \cdot 10^{0}$ & $4.713149 \cdot 10^{-1}$ & $7.728709 \cdot 10^{-1}$ \\
14 & $2.581633 \cdot 10^{0}$ & $1.439926 \cdot 10^{0}$ & $1.954523 \cdot 10^{0}$ & $1.441835 \cdot 10^{0}$ \\
15 & $6.980096 \cdot 10^{-1}$ & $1.365447 \cdot 10^{0}$ & $1.512895 \cdot 10^{0}$ & $6.113783 \cdot 10^{-1}$ \\
16 & $2.216725 \cdot 10^{-1}$ & $1.183319 \cdot 10^{0}$ & $9.590818 \cdot 10^{-2}$ & $3.708697 \cdot 10^{-1}$ \\
17 & $7.949694 \cdot 10^{-2}$ & $1.823265 \cdot 10^{0}$ & $4.303526 \cdot 10^{-1}$ & $1.722231 \cdot 10^{0}$ \\
18 & $1.778746 \cdot 10^{-2}$ & $2.058049 \cdot 10^{-3}$ & $1.200130 \cdot 10^{-2}$ & $5.377238 \cdot 10^{-3}$
\end{tabular}

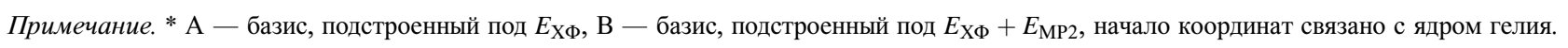

Таблица 3. Дипольные моменты перехода (atomic units) между состояниями $\mathrm{A} \rightarrow \mathrm{X}, \mathrm{C} \rightarrow \mathrm{X}$ и $\mathrm{D} \rightarrow \mathrm{X}$ как функция межъядерного расстояния $R$

\begin{tabular}{c|c|c|c|c|c|c|c|c|c}
\hline \multirow{2}{*}{$R$, bohr } & \multicolumn{3}{|c|}{$\mathrm{A} \rightarrow \mathrm{X}$} & \multicolumn{3}{c|}{$\mathrm{C} \rightarrow \mathrm{X}$} & \multicolumn{3}{c}{$\mathrm{D} \rightarrow \mathrm{X}$} \\
\cline { 2 - 10 } & $\mathrm{A}$ & $\mathrm{B}$ & $\mathrm{KB}[11]$ & $\mathrm{A}$ & $\mathrm{B}$ & $\mathrm{KB}[11]$ & $\mathrm{A}$ & $\mathrm{B}$ & $\mathrm{KB}[11]$ \\
\hline 1.0 & -0.1562 & -0.1588 & -0.1638 & -0.7461 & -0.8075 & -0.8447 & 0.2739 & 0.2704 & 0.2712 \\
1.25 & -0.5479 & -0.5870 & -0.6366 & -0.5202 & -0.5488 & -0.6084 & 0.2120 & 0.2169 & 0.2107 \\
1.4 & -0.5551 & -0.5861 & -0.6299 & -0.4309 & -0.4562 & -0.5077 & 0.2037 & 0.2050 & 0.2129 \\
1.5 & -0.5182 & -0.5489 & -0.5963 & -0.3973 & -0.4341 & -0.4583 & 0.2177 & 0.2010 & 0.2126 \\
1.6 & -0.4878 & -0.4972 & -0.5587 & -0.3471 & -0.3845 & -0.4200 & 0.2073 & 0.2093 & 0.2098 \\
1.75 & -0.4469 & -0.4541 & -0.5047 & -0.3105 & -0.3376 & -0.3781 & 0.1914 & 0.2007 & 0.2041 \\
2.0 & -0.3800 & -0.3979 & -0.4349 & -0.2817 & -0.3006 & -0.3357 & 0.1732 & 0.1878 & 0.1955 \\
\hline СОП**,\% & 11.5 & 7.9 & 0 & 15.1 & 8.5 & 0 & 4.3 & 2.2 & 0
\end{tabular}

Примечание. * ${ }^{*}$ - базис, подстроенный под $E_{\mathrm{X} \Phi}, \mathrm{B}-$ базис, подстроенный под $E_{\mathrm{X} \Phi}+E_{\mathrm{MP} 2}$.

** СОП - средняя относительная погрешность по сравнению с [11].

что при расчете был использован базис, оптимизи-

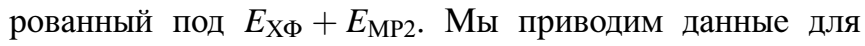
межъядерного расстояния $R=1.5 \mathrm{bohr}$, которое соответствует равновесному расстоянию для первого ВС $\mathrm{A}^{2} \Sigma^{+}$(основное состояние $\mathrm{X}^{2} \Sigma^{+}$является несвязанным, но для полноты мы приводим данные для энергии этого состояния). Как видно из табл. 1, энергия ХФ лишь немного ( 1 mhartree) повышается в результате

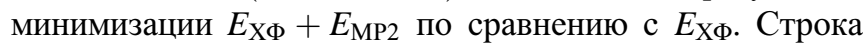
„ТВ“ означает, что значения были получены с учетом энергии корреляции во втором порядке теории возмущений. Для оценки точности мы также приводим результаты расчетов, выполненных методом конфигурационного взаимодействия [11] (строка „КВ““ в таблице).

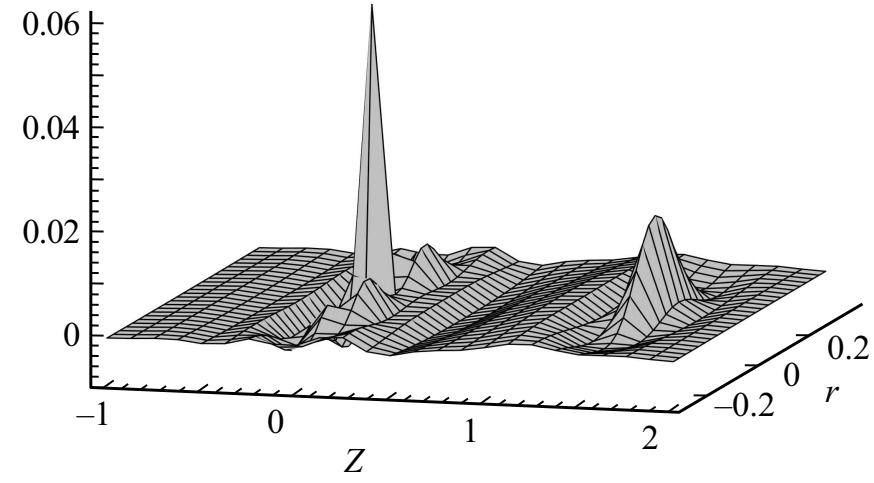

Разность электронных плотностей $\rho_{\text {ХФ }}-\rho_{\text {ХФ+МР2 }}$ (atomic units), которые индуцированы методами ХФ и ХФ + MP2 для ВC $\mathrm{A}^{2} \Sigma^{+}$молекулы НеН при $R=1.5 \mathrm{bohr}$. 
Оптимальные параметры экспонент $\varsigma_{p}$ и центровки $Z_{p}$ базисов, оптимизированных для $E_{\mathrm{X} \Phi}$ и $E_{\mathrm{X} \Phi}+E_{\mathrm{MP2}}$, представлены в табл. 2. Как видно, параметры экспонент второго базиса имеют более диффузный характер в сравнении с первым базисом, адаптирован-

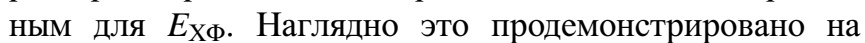
рисунке, где представлена разность электронных плотностей, $\rho(r, Z)$, построенных для базиса ХФ и базиса

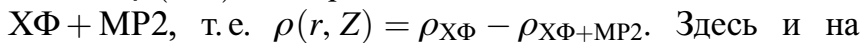
рисунке $r^{2}=x^{2}+y^{2}$ и ось $Z$ направлена вдоль молекулярной оси с началом на ядре Не. Несмотря на сложный характер распределения разности плотностей, можно отметить следующие особенности. Прежде всего видно, что плотность с базисом ХФ больше, чем плотность

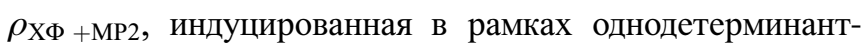
ного приближения (см., в частности, пики на ядрах). С другой стороны, небольшие впадины между ядрами и за ядром Не показывают, что электронная плотность

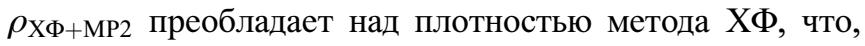
видимо, важно для расчетов ДМП, как это демонстрируется ниже.

В табл. 3 приведены ДМП между состояниями $\mathrm{A}^{2} \Sigma^{+} \rightarrow \mathrm{X}^{2} \Sigma^{+}, \mathrm{C}^{2} \Sigma^{+} \rightarrow \mathrm{X}^{2} \Sigma^{+}$и $\mathrm{D}^{2} \Sigma^{+} \rightarrow \mathrm{X}^{2} \Sigma^{+}$как функция межьядерного расстояния $R$. Для разных типов базисов мы сравниваем значения ДМП между собой и с результатами, полученными в [11] методом конфигурационного взаимодействия с большим количеством опорных конфигураций. Из таблицы видно, что базис, подстроенный под $E_{\mathrm{X \Phi}}+E_{\mathrm{MP2}}$, обеспечивает согласие с высокоточными расчетами, лучшее, чем базис, полученный минимизацией энергии ХФ. При этом средняя относительная погрешность уменьшается почти в два раза для всех рассматриваемых переходов. Это означает, что базис, который неявно отражает корреляционные эффекты, более эффективен для расчетов ДМП, чем базис, оптимизированный для энергии ХФ. Другими словами, предварительные результаты расчетов показывают, что для получения корректных ДМП-результатов следует использовать базисные наборы, которые частично приспособлены для расчета корреляционных эффектов.

\section{Список литературы}

[1] Wilson S. // New Methods in Quantum Theor/ Ed. by Tsipis C.A., Popov V.S., Herschbach D.R., Avery J.S. 1996. P. 437-461.

[2] Jensen F. // J. Chem. Phys. 1999. V. 110. N 14. P. 6601.

[3] Mitin A.V. // Phys. Rev. A. 2000. V. 62. P. 010501.

[4] Glushkov V.N., Kobus J., Wilson S. // J. Phys. B. 2008. V. 41. P. 205102.

[5] Hylleraas E.A. // Z. Phys. 1930. V. 65. P. 209.

[6] Ребане Т.К. // Опт. и спектр. 1989. Т. 66. Вып. 2. С. 484.

[7] Ребане Т.К. // ТЭХ. 1985. Т. 21. C. 346.

[8] Shull H., Löwdin P.-O. // Phys. Rev. 1958. V. 110. P. 1466.

[9] Sarpal B.K., Tennyson J. // J. Phys. B. 1992. V. 25. P. L49.

[10] Tennyson J. // J. Mol. Spectrosc. 2014. V. 298. P. 1.

[11] Theodorakopoulos G., Petsalakis I.D., Nicolaides C.A., Buenker R.J. // J. Phys. B. 1987. V. 20. P. 2339.
[12] Petsalakis I.D., Theodorakopoulos G., Nicolaides C.A., Buenker R.J. // J. Phys. B. 1987. V. 20. P. 5959.

[13] Gurin V.S., Korolkov M.V. http://arxiv.org://arXiv: 2014-1412.5999[physics.chem-ph].

[14] Minaev B.F., Telyatnik L.G. // Opt. Spectrosc. 2001. V. 91. N 4. P. 883.

[15] Knowles P.J., Werner H-J., Hay P.J., Cartwright D.C. // J. Chem. Phys. 1988. V. 89. P. 7334.

[16] Henneker W.H., Popkie H.E. // J. Chem. Phys. 1971. V. 54. P. 1763.

[17] Morokuma K., Iwata S. // Chem. Phys. Lett. 1972. V. 16. P. 195.

[18] Davidson E.R., Stenkamp L.Z. // Int. J. Quant. Chem. (Symp). 1976. V. 10. P. 21.

[19] Murakhtanov V.V., Mazalov L.N., Guzhavina T.I. // Zh. Strukt. Khim. 1981. V. 22. P. 22.

[20] Cheglokov E.I., Lirmak Yu.M., Terpugova A.F. // Izv. Vyssh. Uchebn. Zaved. Fiz. 1985. N 10. P. 55.

[21] Assfeld X., Rivail J.-L. // Chem. Phys. Lett. 1996. V. 263. P. 100.

[22] Glushkov V.N. // Chem. Phys. Lett. 1998. V. 287. P. 189.

[23] Möller C., Plesset M.S. // Phys. Rev. 1934. V. 46. P. 618.

[24] Glushkov V.N., Gidopoulos N., Wilson S. // Frontiers in Quantum Systems in Chemistry and Physics. Progress in Theoretical Chemistry and Physics / Ed. by Wilson S., Grout P.J., Delgado-Barrio G., Maruani J., Piecuch P. Dordrecht: Springer, 2008. Pt. 2. P. 451.

[25] Глушков В.Н. // Опт. и спектр. 2015. Т. 119. № 1. С. 1.; Glushkov V.N. // Opt. Spectrosc. 2015. V. 119. N 1. P. 1.

[26] Glushkov V.N., Assfeld X. // Theor. Chem. Acc. 2016. V. 135. P. 3.

[27] Glushkov V.N. // J. Math. Chem. 2002. V. 31. P. 91.

[28] McWeeny R., Sutcliffe B.T. Methods of Molecular Quantum Mechanics. NY.: Academic Press, 1976. 